\title{
Meta
}

Journal des traducteurs

Translators' Journal

\section{HURTADO ALBIR, Amparo (1990) : La notion de fidélité en traduction, Paris, Didier Érudition, 236 p.}

\section{Jeanne Dancette}

Volume 38, numéro 1, mars 1993

La traduction et l'interprétation dans le nord du Canada

Translation and Interpretation in Northen Canada

URI : https://id.erudit.org/iderudit/002451ar

DOI : https://doi.org/10.7202/002451ar

Aller au sommaire du numéro

Éditeur(s)

Les Presses de l'Université de Montréal

ISSN

0026-0452 (imprimé)

1492-1421 (numérique)

Découvrir la revue

Citer ce compte rendu

Dancette, J. (1993). Compte rendu de [HURTADO ALBIR, Amparo (1990) : La notion de fidélité en traduction, Paris, Didier Érudition, 236 p.] Meta, 38(1),

140-143. https://doi.org/10.7202/002451ar

Ce document est protégé par la loi sur le droit d'auteur. L'utilisation des services d'Érudit (y compris la reproduction) est assujettie à sa politique d'utilisation que vous pouvez consulter en ligne.

https://apropos.erudit.org/fr/usagers/politique-dutilisation/ 
TURTADO ALBIR, Amparo (1990): La notion de fidélité en traduction, Paris, Didier Érudition, 236 p.

Le livre, le $5^{\mathrm{e}}$ de la collection «traductologie», s'attaque à un problème fondamental. La notion de la fidélité en traduction est, en effet, une sorte de mise au carré de l'épineuse question de l'expression de la pensée humaine par le langage à cause du double circuit de la traduction qui va de l'émetteur au destinataire de la traduction en passant par le traducteur lui-même. De fait, la question a déjà été abordée de multiples façons, dans différentes disciplines. D'une part, les linguistes, les informaticiens travaillant dans le domaine de la traduction automatique, les pédagogues et les examinateurs de traduction prennent généralement la fidélité comme critère premier de la qualité de la traduction et la définissent grosso modo comme étant l'adéquation au sens. D'autre part, les courants littéraires, religieux, philosophiques, anthropologiques s'interrogent, eux aussi, sur la question, en cherchant à voir comment la fidélité s'exprime dans un poème, un psaume, une inscription, et quel est le rapport forme-sens qui rend la traduction fidèle au texte de départ. 
Toutefois, des questions anciennes restent encore sans réponse: la fidélité définitelle la valeur de la traduction, le sens prime-t-il sur la forme, quel est le rapport sensforme qui rend compte de la fidélité ? De plus, si la fidélité est une notion qui s'attache, pensons-nous, principalement au produit, elle est intimement liée à celle du choix des moyens linguistiques qui permettent de l'exprimer. À cet égard, la discussion réactive les débats littéralité ou liberté, transcodage ou adaptation (commentaire).

Le livre d'Hurtado Albir se situe donc au cour des nombreuses questions qui ont, de tous temps, enflammé les passions parmi les traducteurs. Cependant, malgré son titre très ambitieux, il apportera au mieux quelques éclaircissements plutôt que des réponses, et on ne saurait y chercher une analyse très poussée ou très nouvelle des nombreux concepts évoqués. Précisons d'emblée que l'ouvrage est solidement ancré dans le courant de l'école interprétative de la traduction développé par D. Seleskovitch et M. Lederer. Reprendre les thèmes du livre revient à rappeler les grandes lignes de ce courant.

Dans une première partie, Hurtado Albir définit la fidélité comme étant la fidélité au sens et non à la forme; elle retrace rapidement les avatars de la notion dans l'Histoire, précisant que le terme a très souvent été compris comme synonyme de «littéralité» par opposition à «traduction libre».

L'auteur s'insère dans le débat traduction littérale - traduction libre pour dire qu'il s'agit d'un faux débat, puisque ni l'une ni l'autre ne peut exprimer la fidélité qui ne sera bien servie que par la méthode dite interprétative, même si elle reconnaît que parfois la traduction littérale peut être fidèle au sens ou, à d'autres moments, que la traduction libre peut être une bonne solution, selon les circonstances et les intentions du traducteur.

Dans la tradition de l'école interprétative qui a eu le mérite de mettre l'accent sur la démarche de la traduction, l'auteur consacre un chapitre entier au processus traductionnel et revient inlassablement sur la méthode interprétative. La fidélité, dit-elle, est le résultat de cette méthode, le processus de traduction est essentiellement interprétatif. Comme ses prédécesseurs (Seleskovitch, Lederer), elle s'appuie sur les travaux de J. Barbizet: «Le souvenir cognitif qu'est le sens serait ainsi constitué par une organisation neuronique (les métacircuits), évidemmment sous forme non-verbale» (p. 55); ainsi que sur les travaux de $\mathbb{B}$. Pottier sur le «mouvement du vouloir dire», mouvement non verbal. Elle reprend également les travaux de J. Delisle sur le parcours de la compréhension et les compléments cognitifs de la traduction.

Dans ce cadre, on ne sera donc pas surpris de trouver cette définition du sens: «synthèse non-verbale résultant du processus opéré par l'individu» (p. 61). Le sens est défini par le «"sens compris" comme résultat du processus de compréhension» (p. 61). De cette «dissociation effectuée entre la forme linguistique et le sens» (p. 61) découle le caractère dynamique du sens, qui doit se construire et se reconstruire en permanence dans le discours et par chaque individu. «Le sens est donc imprévisible et infini.» (p. 75)

Cette première partie théorique contient, en plus d'affirmations souvent trop catégoriques, des contradictions flagrantes. La plus gênante est peut-être la difficulté à concilier cette conception très relative du sens, non linguistique et psychologisante, avec le concept du «sens comme invariant» auquel le chapitre 3 est consacré, ou avec l'idée très normative que «le traducteur doit comprendre le texte original pour exprimer le même sens avec les moyens d'une autre langue» (p. 70) (c'est nous qui soulignons).

Cela dit, l'auteur rappelle quelques leçons importantes pour l'étude de la méthode de la traduction. «On ne comprend pas un texte (ou même une phrase) quand on en a retenu tous les mots, mais quand on en a fait une synthèse, à travers l'élaboration mentale de la compréhension, qui n'est plus verbale» (p. 69). Ce genre d'affirmation va dans le sens des travaux des chercheurs en linguistique textuelle et en psycholinguistique, tels Van Dijk, Kintsch et Le Ny, sur la compréhension de texte et le rôle de la mémoire. 
À propos de la question du sens comme «invariant», l'auteur essaie d'apporter des précisions par le biais de plusieurs paires d'opposition.

1) Signification - sens. La signification, dit-elle, concerne les concepts qui s'attachent au signifiant; le sens «concerne l'acte de parole et il met en jeu la signification actualisée par le contexte (verbal et non verbal).» (p. 74) Notons au passage le glissement: le sens est la signification actualisée, mais qu'est-ce que la signification?

2) Information - sens. L'information, invariable, quelle que soit la forme du message (poétique ou non), intervient dans la construction du sens mais ne doit pas être confondue avec le sens. Ainsi «un poème et un récit peuvent transmettre une même information tout en ayant un sens différent» (p. 76). On regrette que la question de ce que nous pourrions appeler le sens poétique ne soit pas plus développée.

3) L'effet, l'intention de l'auteur (effet voulu), le style, la connotation et l'implicite sont d'autres éléments qui interviennent dans la construction du sens.

L'auteur se démarque de Nida et de Taber qui dissocient la forme du sens: «Si le style change, dit-elle, le sens change lui aussi en raison de cette union indissociable.» (p. 80) Si cette formulation recouvre une certaine évidence, elle gagnerait à être nuancée, en distinguant, par exemple, le sens et l'effet de sens. Prise comme telle, elle conduit à nier l'existence de phrases synonymes; or s'il n'y a pas de synonymie de phrases (à l'intérieur d'une même langue), comment peut-on espérer que la traduction pourra exprimer le «même sens» d'une langue à l'autre?

Définissant le sens dans le cadre de la communication, Hurtado Albir présente des tableaux sur «le circuit du sens» et sur la «fidélité au sens», qui malgré leur caractère simplificateur ont une certaine valeur explicative. «Pour que l'acte de parole soit réussi (...), il faut que le sens compris du récepteur soit égal au vouloir dire de l'émetteur.» (p. 89) C'est dans cette équation que réside la définition de la «fidélité dans la communication unilingue», d'où découle la définition de la fidélité en traduction, «adéquation du sens compris du traducteur avec le vouloir dire de l'auteur et adéquation du sens compris du destinataire de la traduction avec celui du texte original.» (p. 93)

Nous nous attendions à ce que l'auteur aborde la question de l'évaluation ou de la mesure de la fidélité au moment où elle introduit la notion d' «équivalence de sens» sur laquelle est fondée l'identité de sens. Malheureusement, là encore, elle parle plus de méthode (interprétative) que de produit, et ce développement, pour aussi intéressant (si ce n'est nouveau) qu'il soit, est peu pertinent pour l'analyse de la fidélité. L'équivalence est, en effet, vue comme une création continuelle, éphémère et inédite.

Le problème réside peut-être dans le fait que l'auteur confond fidélité (au sens) et réussite de l'acte de communication spécifique qu'est la traduction quand elle définit les trois paramètres de la fidélité: fidélité au vouloir dire de l'auteur, fidélité à la langue d'arrivée et fidélité au destinataire. Cette confusion la conduit à écrire qu'«une traduction qui n'est pas claire pour son destinataire ou qui présente des erreurs de langue n'est pas une traduction fidèle au sens (p. 118)».

La deuxième partie de l'ouvrage, intitulée «Les dimensions de la fidélité», s'appuie sur un corpus de textes bien choisis: les différentes traductions espagnoles d'extraits de classiques français, dont Le Contrat social, L'esprit des lois, Gargantua, La cigale et la fourmi, La mauvaise réputation, Le fils d'Astérix. Les observations sur les écarts d'une traduction à l'autre par rapport au texte original sont fort pertinentes et enrichissent le répertoire de textes analysés sur lesquels nous pouvons échafauder une théorie de la traduction. L'auteur les utilise pour dégager les facteurs qui rendent la fidélité plus difficile à établir : la subjectivité, l'historicité et la fonctionnalité. 
Sur le plan de la forme, nous devons malheureusement signaler que l'ouvrage comporte de nombreuses fautes de frappe et qu'il aurait gagné à faire l'objet d'une révision typographique plus serrée. Un tableau, par exemple, est imprimé à l'envers (p. 65), ce qui rend son décodage difficile quand on n'a pas de miroir sous la main. Verres transparents, verres colorants, faut-il ajouter le miroir à la panoplie du traducteur?

Pour conclure, nous dirons que le livre, malgré ses lacunes et faiblesses, mérite une place dans la bibliothèque du traducteur, pour les trois raisons suivantes: il traite d'une question importante en traductologie, contribue à mieux faire comprendre et apprécier la portée et les limites de la théorie interprétative et, enfin, présente une application à un ensemble de textes variés, dont la difficulté même pose un défi. À lire avec un esprit critique.

JEANNE DANCETTE Université de Montréal, Montréal, Canada 